\title{
Exploration on Internationalization of Curriculum Structural Analysis of Organic Compounds
}

\author{
Hongqi $\mathrm{Li}^{1, \mathrm{a}}$ and Yanxi Song ${ }^{2, b}$ \\ ${ }^{1}$ College of Chemistry, Chemical Engineering and Biotechnology, Donghua University, \\ Shanghai 201620, China \\ ${ }^{2}$ School of Environmental Science and Engineering, Donghua University, \\ Shanghai 201620, China \\ ahongqili@dhu.edu.cn, bsyx@dhu.edu.cn
}

Keywords: organic compounds; structural analysis; curriculum internationalization.

\begin{abstract}
Considering the international generality of knowledge related to structural analysis of organic compounds, we tried to explore internationalization of curriculum Structural Analysis of Organic Compounds. Intercultural and internationalized contents were added to current curriculum by the infusion way. The teaching method became diversified and more internationalized. Preliminary exploration on establishment of practice course was conducted. Improvement in teaching effect was achieved by the internationalization of the curriculum.
\end{abstract}

\section{Introduction}

With the rapid development in globalization of economy and culture, internationalization has become one of the main tendencies in higher education reform and development in China since 1990s. Internationalization of university curricula was especially attractive in the respect. It was generally defined to be a process of development and establishment of a foreign language curriculum or an international local research curriculum and a process of universalization of disciplines. Specifically speaking the internationalization of curricula mainly means the internationalizing tendency in the guidelines of the curricula establishment, in the specific curricula contents, and in the implementation ways, with the ultimate aim to cultivate talents who exhibit international vision and international communication ability in the globalization background ${ }^{[1-4]}$.

Spectroscopy serves as an important methodology for identification of organic compounds and determination of their chemical structures. It is combined tightly with electronics and computer science thus becomes vital in structural analysis and has been extensively applied to varied disciplines such as chemistry, chemical engineering, pharmacology, material science and biology. The curriculum Structural Analysis of Organic Compounds involves mainly in fundamental principles, characteristics, laws and spectral resolution techniques of spectroscopy including ultraviolet-visible (UV-Vis), infrared (IR) and nuclear magnetic resonance (NMR) spectroscopy, and mass spectrometry (MS). The curriculum has been a special basis course for students of varied majors, for example, chemistry, life science, material science, environment science, medicine and food science in universities or colleges. The main teaching aim of the curriculum is to enable the students to grasp the fundamental techniques and methods and to cultivate the students' ability of deducing the structures of organic compounds by using their spectroscopic knowledge.

Knowledge related to chemistry especially structural analysis of organic compounds is of international common. Therefore the curriculum Structural Analysis of Organic Compounds is fit for internationalization which will be beneficial to students in the following aspects: improvement in their specialized English level, improved understanding of fundamental techniques and methods for structural analysis of organic compounds in the more common sense, better adaptability to English literature and exercises and lectures held by foreign experts, improvement in communication with 
foreign scholars and experts on specialized fields, broadening their international horizon, grasp of modern education thoughts and the world's leading knowledge in related fields ${ }^{[5-8]}$. Hereinafter we will introduce the preliminary exploration result from our internationalization of master curriculum Structural Analysis of Organic Compounds and our teaching thoughts.

\section{Shortcomings in the curriculum}

The contents in the curriculum Structural Analysis of Organic Compounds are abstract and cumbersome to beginners. They feel difficult to keep firmly in mind while easy to become confused by these contents. Even they may learn the fundamental principles and contents related to the above-mentioned spectroscopy their ability to recognize and resolve spectra and eventually determine the structures of unknown organic compounds by using their spectroscopic knowledge proves insufficient. The students in the class are from different majors including organic chemistry, applied chemistry and textile chemistry and dyeing and finishing engineering. They show vast differences in their learning foundation. All of them show high enthusiasm for the curriculum study. Most of them can master the fundamental techniques and methods for structural analysis of organic compounds through the curriculum study and some of them gain the ability to resolve the chemical structures of organic compounds by using their knowledge. However, many students are poor in specialized English and can not understand the contents in English literature and the lectures by foreign experts. Their international communication ability is very poor. On the other hand, the foreigner students in the class are weak in Chinese thus can not understand the contents taught in Chinese. They need to be provided with English textbooks, references, coursewares and exercises.

\section{Optimization and internationalization of curriculum contents}

To realize internationalization and universalization of disciplines the writer of textbooks must stand on the frontier of the subject, have global horizons and achieve mastery through a comprehensive study of the subject. He should not only know the developing history of the subject but also master its current research status and future developing prospects. We adopted infusion method for internationalization of the curriculum contents. That is to say, intercultural and internationalized contents were added to the current curriculum. Foreign typical examples were selected to enrich the curriculum. Apart from the in-use Chinese textbooks (for example, Structural Analysis of Organic Compounds edited by Jianping Zou, Lu Wang and Runsheng Zeng and published by Science Press in 2005 and Spectroscopic Analysis edited by Shuangyan Huan and published by China Textile Press in 2008), a part of contents and typical examples from foreign textbooks (such as Spectrometric Identification of Organic Compounds seventh edition edited by Robert M. Silverstein, Francis X. Webster and David J. Kiemle and published by John Wiley \& Sons, Inc. and Organic Spectroscopic Analysis edited by R. J. Anderson, D. J. Bendell and P. W. Groundwater and published by The Royal Society of Chemistry) were added as the curriculum contents. Meanwhile a tutoring website of the curriculum was in construction in order that the students could read the electronic teaching plan, multimedia courseware and exercises for after-class study. In the puzzle-solving aspect, we intend to establish a blog forum in order that the students can access to replies from the teacher at any time.

\section{Diversification and internationalization of teaching methods}

Teaching methods have a vital impact on teaching effect. We conducted the following exploration and tests and propose suggestions for further exploration.

(1) Addition of flash to teaching. Addition of some flash slides to PPT (Power Point) was a compensation for the boring description in the textbooks. For instance, in the teaching of IR spectra, we could teach and explain the vibrational modes of molecules by flash with bright colors and special visual effects. Thus the dull molecular structures and various vibrational modes looked interesting, which greatly stimulated the interest of the students. The classroom atmosphere turned active and 
communication between the teacher and students was enhanced. Abstract knowledge seems lively and interesting to students.

(2) Addition of English coursewares. In the process of courseware making some Chinese coursewares were necessary considering the receptivity of the students. Addition of English and bilingual coursewares could not only benefit foreigner students who were poor in Chinese but also help Chinese students learning specialized English which would be of help for them to search and read specialized literature after they enter the laboratory.

(3) Arrangement of lectures by foreigner experts and scholars. If the students can participate in a series of specialized lectures and seminars by foreigner experts and scholars their English level, specialized ability and internationalization ability may be improved.

\section{Design and trial of addition of practice course}

Addition of practice stage is helpful for students to improve their practical operative ability and ability to resolve structures of organic compounds by using their knowledge. We explored to add several experiments to the curriculum, combining the "entering laboratory" activity organized by the students, to provide students with watching, studying and operating by themselves UV absorption spectrophotometer, Fourier-transform IR spectrometer and NMR spectrometer. By using UV spectrophotometer to determine UV spectra of different types of compounds, the students mastered the whole determination procedure including selection of solvent, preparation of solution and measurement of samples. They acquired more perceptual knowledge on relationship between UV spectra and conjugation of structures and conjugation degree of compounds. Our university and college provided students with good experimental opportunities for carrying out IR spectra determination. Therefore they could measure IR spectra of compounds conveniently. Students majoring in organic chemistry, applied chemistry or textile chemistry and dyeing and finishing engineering would use IR spectra for structural characterization in their forthcoming graduation dissertation work so they showed high interest and motivation for experiments on IR spectra measurement. Through this practical training the students got relatively profound understanding on how to use IR spectra to determine structures of compounds and to identify the presence or absence of a specific functional group in a compound. They also gained knowledge on monitoring a reaction and determining composition of samples by using IR spectra. Students majoring in organic chemistry were provided with further training in using NMR spectrometer for structure identification of organic compounds.

On the basis of preliminary exploration of carrying out spectral practice course, we design to introduce two comprehensive organic spectroscopic experiments into the curriculum. One involves in synthetic reactions, separation method, purification techniques, spectral measurement, structural characterization and identification of new compounds. The other is an experiment for structural analysis of a sample, aiming at enabling the students to master the whole procedure including collection of fundamental information of the sample, measurement of the basic properties of the sample, components separation and identification of compositions in the sample, and to improve their comprehensive practical ability for organic spectrometric analysis.

\section{Summary}

Internationalization of university curricula, as one of main tendencies in higher education reform and development in China and the product of globalization of economy and culture, has become a focus in education. How to conduct the internationalization of university curricula? Which curricula are fit for internationalization prior to other curricula? What are the differences between curricula internationalization and bilingual teaching? Many related issues are still needed deeper and more systematic study and exploration. Considering that knowledge related to structural analysis of organic compounds is of international common we made preliminary exploration on internationalization of curriculum Structural Analysis of Organic Compounds in order to improve the 
teaching effect and to throw a sprat to catch a whale. We adopted infusion method for internationalization of the curriculum contents, adding intercultural and international contents, foreign typical examples, contents in English textbooks and lectures by foreigner experts and scholars to the current curriculum. Diversification and internationalization of teaching methods were explored. In the future further study and verification are needed on several issues such as how to set up practice courses more effectively and how to make the examination more internationalized.

\section{References}

[1] Q.-J. Ouyang: Theory Research No. 3 (2012), p. 160

[2] Y. Jiang, Z. Sun and H. Zhang: Open Education Research Vol. 19 No. 4 (2013), p. 74

[3] S. Liu and Y. Tao: Journal of World Education No. 20 (2013), p. 56

[4] W. Zhao and H. Zhao: Magnificent Writing No. 2 (2014), p. 227

[5] D. Liang: Popular Science \& Technology Vol. 14 No. 12 (2012), p. 121

[6] C.-X. Wang, C.-L. Xu, G.-Y. Yang and S.-F. Fang: Chinese Journal of Spectroscopy Laboratory Vol. 29 No. 4 (2012), p. 2419

[7] X. Tian, J. Feng, X. Zhang, H. Wu and X. Zhang: Science \& Technology Information No. 19 (2013), p. 206

[8] J. Guo, Q. Guo, L. Li, G. Yan, X. Yu, F. Du and Q. Zhang: Chemical Industry Times Vol. 27 No. 9 (2013), p. 52 